\title{
Epidemiologia do Araneísmo de 2005 a 2013 no Centro de Assistência Toxicológica, João Pessoa-PB, Brasil
}

\author{
Epidemiology of Araneism between 2005 and 2013 at the Toxicological Assistance \\ Center, João Pessoa-PB, Brazil
}

\author{
VANESSA MEDEIROS DANÓBREGA ${ }^{1}$ \\ SAEMMY GRASIELY ESTRELA DE ALBUQUERQUE ${ }^{1}$ \\ MAYARA MUNIZ DIAS RODRIGUES ${ }^{1}$ \\ IRIS MARIANADA COSTABARROS ${ }^{2}$ \\ MARIA DE FÁTIMA LEANDRO MARQUES ${ }^{3}$
}

\section{RESUMO}

Registros de araneísmo no nordeste do Brasil são escassos e, possivelmente, subestimam a verdadeira situação de risco. Objetivo: analisar o perfil clínico-epidemiológico do araneísmo no período de 2005 a 2013 notificados no Centro de Assistência Toxicológica da Paraíba. Materiais e métodos: estudo descritivo com dados coletados no banco de dados do Centro de Assistência Toxicológica da Paraíba e em suas fichas de notificação dos acidentes. Resultados: Ocorreram 484 notificações de acidentes com aranhas, nos quais dos 202 casos com identificação de gênero, prevaleceu os acidentes com caranguejeira $(57,9 \%)$ e Loxosceles $(33,2 \%)$. Quanto à sazonalidade, os meses com maior número de ocorrências foram agosto, março, janeiro e junho, respectivamente. A maioria dos acidentes ocorreu em zona urbana; o sexo feminino e a faixa etária entre os 21 aos 30 anos foram os mais acometidos; e os membros superiores e os inferiores foram os locais mais atingidos. As principais manifestações clínicas foram dor, eritema, edema e prurido. Conclusão: Os resultados indicam que a prevalência do araneísmo pode apresentar relação com o urbanismo, não ocorreu predominância de casos graves.

\section{DESCRITORES}

Aranhas. Animais Peçonhentos. Epidemiologia descritiva. Estudos retrospectivos. Prevenção de Acidentes. Toxicologia.

\begin{abstract}
Araneism records in northeastern Brazil are scarce and possibly underestimate the actual risk situation. Objective: to analyze the clinical and epidemiological profile of araneism during the 2005-2013 period reported in the Toxicological Assistance Center of Paraiba. Material and Methods: This is a descriptive study using data collected from the database of the Toxicological Assistance Center of Paraíba and from accident notification files. Results: There were 484 notifications of accidents with spiders, amongst which only 202 cases identified the spider genus, with a high prevalence of Brazilian spider "caranguejeira" (57.9\%) and Loxosceles $(33.2 \%)$. Regarding seasonality, August, March, January and June, were the months with the highest number of occurrences, respectively. Most accidents occurred in urban areas; females and 21-30 age groups were the most affected ones; and the upper and lower limbs were the most affected parts of the body. The main clinical manifestations were pain, redness, swelling and itching. Conclusion: The results indicate that the prevalence of araneism can be related to urbanism There was no predominance of severe cases.
\end{abstract}

\section{DESCRIPTORS}

Spiders. Animals, Poisonous. Epidemiology, Descriptive. Retrospective Studies. Accident Prevention. Toxicology.

\footnotetext{
Doutoranda do Programa de Pós-Graduação em Enfermagem da Universidade Federal da Paraíba, João Pessoa (PB), Brasil.

2 Enfermeira. Diretora Administrativa Financeira no Distrito Sanitário II na Secretaria Municipal de Saúde de João Pessoa, João Pessoa (PB), Brasil.

3 Enfermeira. Membro do Comitê de Ética em Pesquisa do Centro de Ciências da Saúde da Universidade Federal da Paraíba, João Pessoa (PB),
} Brasil. 
Ni o mundo existem cerca de 35.000 espécies de aranhas, sendo que apenas cerca de 20 a 30 espécies são perigosas para o homem e podem causar envenenamento significativo. No Brasil, as espécies causadoras de acidentes de relevância médica pertencem aos gêneros Phoneutria, Loxosceles e Latrodectus ${ }^{1}$.

$\mathrm{Na}$ maioria das notificações dos acidentes a espécie de aranha não é informada, considerando que muitos dos animais levados pelas vítimas chegam geralmente amassados, incompletos ou podres devido ao impulso das vítimas de matá-los e da delicadeza de suas estruturas, dificultando ou impossibilitando sua identificação².

O araneísmo, apesar de sua importância médica, é relativamente negligenciado quando comparado ao acidente ofídico. Contudo, ainda se configura como um problema de saúde pública, em especial, nas regiões tropicais do mundo ${ }^{3}$. No nordeste do Brasil, os registros de acidentes por aranhas, são escassos e, possivelmente, subestimam a verdadeira situação de risco ${ }^{4}$, apesar de os dados dos acidentes por animais peçonhentos no Brasil serem reunidos por diversos sistemas de notificação. Esse fato decorre da dificuldade de acesso aos serviços especializados em muitos municípios brasileiros, o que contribui com a subnotificação e não reconhecimento da real dimensão desse problema ${ }^{5}$.

Diante do exposto, o presente estudo teve como objetivo analisar o perfil clínico-epidemiológico dos acidentes por aranhas no período de 2005 a 2013 notificados no Centro de Assistência Toxicológica da Paraíba (CEATOX - PB), localizado no município de João Pessoa - Paraíba.

\section{MATERIAIS E MÉTODOS}

Estudo descritivo e retrospectivo, com abordagem quantitativa de informações sobre acidentes com aranhas ocorridos no município de João Pessoa, no período de 2005 a 2013, e notificados no Centro de Assistência Toxicológica da Paraíba (CEATOX-PB), localizado no Hospital Universitário Lauro Wanderley (HULW).

O serviço onde foram coletados os dados atende casos pelo telefone 0800-7226001 em que a própria vítima ou mesmo um profissional de saúde ligam para saber a melhor conduta a ser implementada diante da sintomatologia apresentada após o acidente. A vítima ao ligar para o CEATOX-PB é orientada a procurar o serviço para melhor avaliação do caso. Os casos de araneísmo que chegam ao serviço são avaliados pelos plantonistas do CEATOX e, caso necessário, segue ao setor de doenças tropicais para consulta médica e realização dos procedimentos necessários.

$\mathrm{O}$ estudo respeitou o que rege a Resolução $N^{\circ} 466 / 12^{6}$ durante a coleta de dados, realizada no período de outubro de 2013 a janeiro de 2014, utilizando-se de duas fontes: o banco de dados do CEATOX-PB e as fichas de acidentes por animais peçonhentos. Inicialmente, com banco de dados dos casos notificados no CEATOX-PB, que foi iniciado no ano de 2006, traçou-se o perfil das notificações no Centro. Posteriormente, foram analisadas nas fichas de notificação de acidentes as seguintes variáveis: mês de ocorrência do acidente, sexo e faixa etária das vítimas, local do corpo atingido; zona de ocorrência, gênero da aranha envolvida, tempo decorrido da picada, manifestações clínicas sistêmicas e locais apresentadas e o tratamento prescrito para o araneísmo.

Os dados foram analisados mediante estatística descritiva com o uso do software Statistical Package for the Social Sciences - SPSS 20.0. O projeto foi aprovado pelo Comitê de Ética em Pesquisa - HULW sob o Protocolo n ${ }^{\circ} 131 / 08$ e CAAE (Certificado de Apresentação para Apreciação Ética) $n^{\circ}$ 0029.0.126.000-11.

\section{RESULTADOS}

No período de 01 de janeiro de 2006 a 31 de dezembro de 2013 foi registrado no CEATOXPB 19.775 notificações, das quais o escorpionismo teve maior destaque com $69,1 \%$ dos casos. Posteriormente apresentam-se os acidentes por outros animais peçonhentos com $5,3 \%$; ofidismo $5 \%$; medicamentos $3,4 \%$; araneísmo $2,4 \%$; produtos químicos industriais e domissanitários $1,5 \%$ cada; raticidas $1,4 \%$; e agrotóxicos de uso agrícola, agrotóxico de uso doméstico, produtos veterinários, cosméticos, metais, drogas de abuso, plantas, alimentos e outros animais não peçonhentos com menos de $1 \%$ de notificações cada. Os casos ocorridos com outros tipos de animais não identificados na ficha de notificação resultaram em $6,2 \%$.

Os acidentes ocasionados por aranhas e notificados no CEATOX estão especificados na Tabela 1. 


\begin{tabular}{|c|c|c|c|c|c|c|c|c|c|c|}
\hline \multirow{2}{*}{$\begin{array}{l}\text { Gênero da } \\
\text { aranha }\end{array}$} & \multicolumn{10}{|c|}{ Ano de ocorrência } \\
\hline & 2005 & 2006 & 2007 & 2008 & 2009 & 2010 & 2011 & 2012 & 2013 & TOTAL \\
\hline Caranguejeira & 20 & 08 & 11 & 11 & 18 & 16 & 14 & 12 & 07 & 117 \\
\hline Latrodectus & 0 & 0 & 01 & 01 & 0 & 01 & 0 & 01 & 0 & 04 \\
\hline Loxosceles & 07 & 09 & 10 & 03 & 08 & 13 & 07 & 08 & 02 & 67 \\
\hline Lycosa & 0 & 03 & 04 & 0 & 0 & 01 & 0 & 01 & 0 & 09 \\
\hline Phoneutria & 0 & 0 & 02 & 02 & 0 & 0 & 0 & 0 & 01 & 05 \\
\hline Não identificado & 28 & 27 & 22 & 17 & 36 & 16 & 34 & 35 & 67 & 282 \\
\hline TOTAL & 55 & 47 & 50 & 34 & 62 & 47 & 55 & 57 & 77 & 484 \\
\hline
\end{tabular}

Das 484 notificações de araneísmo (Tabela 1), em $58,3 \%$ destas não foi possível identificar o gênero envolvido. Dos que procuraram o serviço com até 5 horas, $74,4 \%$ dos casos em que houve identificação do gênero, a caranguejeira esteve envolvida. No loxoscelismo $70,1 \%$ das vítimas procuraram atendimento após as 24 horas do ocorrido. Os meses com maior número de ocorrências foram agosto com $12,8 \%$, março com $10,5 \%$ e janeiro e junho com $10,1 \%$ cada.

Dos 9 casos de administração do Soro Anti-aracnídeo (SAA) cinco não foi possível a identificação do gênero envolvido e quatro dos acidentes o gênero envolvido foi o loxosceles, que correspondeu a necessidade de utilização do soro em $6 \%$ dos acidentes ocasionados por esse gênero.

\section{DISCUSSÃO}

Das notificações do CEATOX-PB, verificase ser pequena a porcentagem dos casos de araneísmo, quando comparados com os demais registros. Contudo, o quantitativo de vítimas por aranhas ainda é representativo, apesar da subnotificação por não procura das vítimas para avaliação e registros de casos leves e que decorrem sem grandes alterações.

Dados epidemiológicos de um estudo realizado no município de Santa Catarina, no período de 2007 a 2011, mesmo assim revelaram um aumento gradativo da incidência de casos por araneísmo, sabendo ser predominantes estas notificações nas regiões Sul e Sudeste do país ${ }^{7}$. Constatou-se número elevado de acidentes por aranhas sem a identificação do gênero. Esse fato decorre de, muitas vezes, a vítima não levar o animal que a picou, não saber descrever características que colaborassem com a identificação ou mesmo a impossibilidade da identificação pelo grau de danificação do material levado. Desse modo, vale salientar que os casos registrados de Phoneutria e Latrodectus no CEATOX-PB foram decorrentes da identificação através da sintomatologia apresentada pelas vítimas.

Os acidentes causados por Phoneutria nigriventer, apesar de sua importância clínica, são pouco descritos de forma detalhada na literatura de envenenamento sistêmico, o que do mesmo modo dificulta a confirmação dos seus casos ${ }^{8}$. Quanto aos acidentes envolvendo Latrodectus, estudo ${ }^{9}$ ressalta não existir testes para confirmação do diagnóstico e que a descrição dos casos pode facilitar a identificação e o manejo correto pelo profissional de saúde de acordo com o tipo de envenenamento.

Esses artrópodes peçonhentos refugiamse dentro das residências em decorrência da ocupação urbana desorganizada, com modificação do habitat natural das aranhas, o que faz com que procurem abrigo em domicílios presentes na zona urbana, aumentando sua importância como problema de saúde pública.

A incidência maior do araneísmo ocorreu em mulheres e os membros superiores e inferiores os locais de maior acometimento. Podem-se justificar esses achados devido à maioria dos acidentes ocorrerem no interior das residências, local em que as aranhas refugiam-se e acabam procurando abrigo da luz em sapatos, calças, roupas penduradas, roupas 
Tabela 2: Perfil clínico-epidemiológico do araneísmo registrados no CEATOX-PB no município de João Pessoa no periodo de 2005 a 2013, segundo as variáveis gênero, zona de ocorrência, sexo, faixa etária, local acometido, tempo decorrido, espécie, sinais e sintomas, condutas e medicamentos.

\begin{tabular}{|c|c|c|c|}
\hline Variáveis & Cateqorias & $\mathbf{n}$ & $\%$ \\
\hline \multirow[t]{5}{*}{ Gênero identificado } & Loxosceles & 67 & 33,2 \\
\hline & Caranqueieira & 117 & 57,9 \\
\hline & Phoneutria & 5 & 2,5 \\
\hline & Lactrodectus & 4 & 2,0 \\
\hline & Lycosa & 9 & 4,5 \\
\hline \multirow{3}{*}{ Zona de ocorrência } & Urbana & 365 & 75,4 \\
\hline & Rural & 66 & 13,6 \\
\hline & lanorado & 53 & 11,0 \\
\hline \multirow[t]{2}{*}{ Sexo } & Feminino & 272 & 56,2 \\
\hline & Masculino & 212 & 43,8 \\
\hline \multirow[t]{9}{*}{ Faixa etária } & 0 a 10 anos & 49 & 10,1 \\
\hline & 11 a 20 anos & 68 & 14,0 \\
\hline & 21 a 30 anos & 117 & 24,2 \\
\hline & 31 a 40 anos & 102 & 21,1 \\
\hline & 41 a 50 anos & 89 & 18.4 \\
\hline & 51 a 60 anos & 30 & 6.2 \\
\hline & 61 a 70 anos & 22 & 4.5 \\
\hline & 71 a 80 anos & 4 & 0.8 \\
\hline & 81 ou mais & 3 & 0,6 \\
\hline \multirow[t]{5}{*}{ Local acometido } & Membros superiores & 200 & 41,3 \\
\hline & Membros inferiores & 168 & 34,8 \\
\hline & Tronco/qlúteo & 68 & 14,0 \\
\hline & Cabeça/pescoco & 42 & 8,7 \\
\hline & lanorado & 6 & 1,2 \\
\hline \multirow[t]{4}{*}{ Tempo decorrido } & Menos de 1 hora & 109 & 22,5 \\
\hline & 1 hora até 5 horas & 115 & 23,8 \\
\hline & 5 horas até24 horas & 61 & 12,6 \\
\hline & 24 horas ou mais & 199 & 41,1 \\
\hline \multirow[t]{15}{*}{ Sinais e Sintomas } & Dor & 283 & 58,5 \\
\hline & Ardor & 33 & 6,8 \\
\hline & Bolhas & 58 & 12,0 \\
\hline & Necrose & 20 & 4,1 \\
\hline & Equimose & 17 & 3,5 \\
\hline & Edema & 165 & 34,1 \\
\hline & Dormência & 87 & 18,0 \\
\hline & Exantema & 11 & 2,3 \\
\hline & Eritema & 215 & 44,4 \\
\hline & Prurido & 119 & 24,6 \\
\hline & Cefaleia & 41 & 8.5 \\
\hline & Febre & 40 & 8,3 \\
\hline & Náuseas/Vômitos & 30 & 6,2 \\
\hline & Tontura & 9 & 1,9 \\
\hline & Nenhum & 33 & 6,8 \\
\hline \multirow[t]{7}{*}{ Condutas } & Hidratação & 11 & 2,3 \\
\hline & Limpeza local & 87 & 18,0 \\
\hline & Compressa morna & 27 & 5,6 \\
\hline & Compressa fria & 51 & 10,5 \\
\hline & Observação & 93 & 19,2 \\
\hline & Exames laboratoriais & 16 & 3.3 \\
\hline & Desbridamento & 8 & 1,7 \\
\hline \multirow[t]{6}{*}{ Medicamentos } & Antibiótico & 88 & 18,2 \\
\hline & Corticoide & 152 & 31,4 \\
\hline & Anti-inflamatório & 25 & 5.2 \\
\hline & Analqésico & 108 & 22,3 \\
\hline & Soro Anti-aracnídeo & 9 & 1,9 \\
\hline & Anti-histamínico & 135 & 27,9 \\
\hline
\end{tabular}


de cama e banho, e ao sentir-se comprimida acabam utilizando como forma de defesa a picada ${ }^{3}$.

Dos casos em que houve identificação da aranha, os acidentes causados por aranhas caranguejeiras foram os mais incidentes, apresentando como sinais e sintomas mais frequente dor, eritema, prurido, dormência e edema e com menor frequência queimação local, cefaleia e náuseas. Trata-se de um grupo de menor interesse médico, quando comparados ao Loxosceles e a Phoneutria, por exemplo, porém, os profissionais de saúde devem valorizá-los no intuito de minimizar o sofrimento das pessoas, uma vez que foi constatado que em $50,4 \%$ dos acidentes por caranguejeiras a dor esteve presente.

Quanto ao loxoscelismo, apesar de a literatura afirmar ser mais frequente nas regiões sul e sudeste ${ }^{10}$, no CEATOX-PB ocupou a segunda posição de registros de araneísmo e fato preocupante, em relação a este tipo de acidente, foi que houve demora pela busca dos serviços, o que contribuiu para o surgimento de complicações como necrose em $17,9 \%$ nas vítimas por Loxosceles. Sabe-se que há comprometimento da eficácia da soroterapia para este tipo de acidente, quando se ultrapassa o tempo máximo recomendado de 72 horas.

O retardamento da busca precoce pela população para atendimento nos casos de loxocelismos decorre da picada ser quase indolor, que associada ao desconhecimento dos sinais e sintomas iniciais e da não relação desses primariamente com a picada de aranha, resulta em não valorização do acidente. Desse modo, as vítimas geralmente buscam atendimento somente quando a dor local piora, o que ocorre entre 12 e 36 horas após a picada ${ }^{3}$.

Portanto, percebe-se a necessidade de conscientização das vítimas de araneísmo não postergarem a procura dos serviços de referência para avaliação e início precoce do tratamento correto para cada caso.

$\mathrm{Na}$ área da saúde, a incidência de intoxicações e envenenamentos, no Brasil, como no resto do mundo, constitui um grave problema de saúde pública e para mudar esse quadro, as autoridades responsáveis por este setor precisam de informações fidedignas para o levantamento da prevalência desses casos, subsídio relevante para o planejamento de suas ações ${ }^{11}$.

Observou-se variação nas condutas ou mesmo nos tratamentos implementados para atender as vítimas envolvidas em acidentes com uma mesma espécie. Isso decorre da ausência de protocolos de atendimentos que norteiem os profissionais em relação ao melhor tratamento de acordo com os sinais e sintomas apresentados pelas vítimas de araneísmo.

Nesse serviço, a principal sintomatologia apresentada pelas vítimas foi dor, edema, eritema e prurido, sendo os acidentes classificados como leve e evoluindo para a cura.

\section{CONCLUSÃO}

No período estudado foram registrados 19.775 casos de acidentes no CEATOX-PB, dos quais $484(2,4 \%)$ foram provocados por aranhas. $\mathrm{O}$ sexo mais acometido foi o feminino $(56,2 \%)$ e faixa etária mais atingida foi a de 21 a 30 anos $(24,2 \%)$, houve predominância de casos na área urbana $(75,4 \%)$, dos 202 casos que tiveram identificação do animal $57,9 \%$ corresponderam à caranguejeira; $33,2 \%$ de casos por Loxosceles; $4,5 \%$ Lycosa; $2,5 \%$ Phoneutria e $2 \%$ por Lactrodectus. A maior parte das vítimas foi atendida em até 5 horas $(46,3 \%)$. Os membros superiores foram os mais acometidos com $41,3 \%$ e os principais sintomas foram dor, prurido, dormência e queimação local.

Apesar da subnotificação do araneísmo, o mesmo representou $2,4 \%$ das notificações do CEATOX-PB e compreende-se como imprescindível a necessidade de estudos epidemiológicos neste campo no Brasil, por haver poucas produções científicas sobre essa temática.

\section{REFERÊNCIAS}

1. Instituto Butantan. Material Didático [Internet]. São Paulo; 2007 [acesso em 2014 jun 27] Disponível em: http:// www.butantan.gov.br

2. Lise F, Coutinho SED, Garcia FRM. Características Clínicas do Araneísmo em Crianças e Adolescentes no Município de Chapecó, Estado de Santa Catarina, Brasil. Acta Sci Health Sci. 2006; 28(1):13-6.

3. Chagas FB, D'Agostoni FM, Betrame V. Aspectos epidemiológicos dos acidentes por aranhas no Estado do Rio Grande do Sul, Brasil. Evidência. 2010; 10(1):121-30.

4. Brazil TK, Pinto-Leite CM, Silva LMA, Lira RMS, Brescovit AD. Aranhas de importância médica do Estado da Bahia, Brasil. Gaz. Méd. Bahia. 2009; 79(1):32-7. 
5. Lemos JC, Almeida TD, Fook SML, Paiva AA, Simões MOS. Epidemiologia dos acidentes ofídicos notificados pelo Centro de Assistência e Informação Toxicológica de Campina Grande (Ceatox-CG), Paraíba. Rev. bras. epidemiol. 2009; 12(1):50-9.

6. Brasil. Resolução 466/12 do Conselho Nacional de Saúde/MS Sobre Diretrizes e Normas Regulamentadoras de Pesquisa envolvendo seres humanos. Diário Oficial da União, 2012.

7. Gerência de Vigilância de Zoonoses e Entomologia. Araneísmo. Diretoria de Vigilância Epidemiológica. Secretária de Estado da Saúde. Santa Catarina, Brasil, 2012. Disponível em: http://www.dive.sc.gov.br/ conteudos/zoonoses/animaispec/docs/ Aranhas_Para_profissionais.pdf. Acesso 10 Set. 2014.

8. Medeiros SB, Pereira CDFD, Ribeiro JLS, Gurgel L, Fernandes G, Tourinho FSV. Accidents Caused by Phoneutria Nigriventer: Diagnosis and Nursing Interventions. J. res.: fundam. care. online 2013; 5(4):467-74

9. Almeida Ramb, Ferreira Junior RS, Chaves CR, Barraviera B. Envenomation caused by Latrodectus geometricus in São Paulo state, Brazil: a case report. J. Venom. Anim. Toxins incl. Trop. Dis. 2009; 15(3):56271.
10. Ministério da Saúde (Brasil). Guia de vigilância epidemiológica. 7 ed. Brasília: Ministério da Saúde; 2009. p. $799-801$.

11. Santana Rosane Abdala Lins de, Bochner Rosany, Guimarães Maria Cristina Soares. Sistema nacional de informações tóxico-farmacológicas: o desafio da padronização dos dados. Ciênc. saúde colet 2011; 16(Suppl 1):1191-200.

\section{Correspondência}

Vanessa Medeiros da Nóbrega

Rua Professora Maria Pinheiro de Almeida, 20, apto 109, Castelo Branco I - CEP: 58050-040

João Pessoa - Paraíba - Brasil

nessanobregam@hotmail.com 